\title{
Nondestructive magnetic inspection of spot welding
}

\author{
Gábor Vértesy a and Ivan Tomágo \\ ${ }^{a}$ Centre for Energy Research, Institute of Technical Physics and Materials Science, \\ Hungarian Academy of Sciences, Budapest, Hungary \\ ${ }^{\mathrm{b}}$ Institute of Physics ASCR, Prague, Czech Republic
}

\begin{abstract}
A method of Magnetic adaptive testing (MAT), which is based on systematic measurement and evaluation of minor hysteresis loops is suggested as simple, reliable and sensitive characterization of welded spots. The method is based on the fact that the welding process has a great influence on the microstructure of the welded area, which modifies the magnetic properties. A series of welded spots, produced by different energy of welding current on carbody sheets was investigated. Good correlation was found between the result of destructive characterization and nondestructive magnetic descriptors.
\end{abstract}

Keywords: Spot welding, Magnetic NDT, Magnetic adaptive testing

Corresponding author: Gábor Vértesy

E-mail address: vertesy.gabor@energia.mta.hu

Telephone numbers: +36-1-3922677, +36-20-4466329

ORCID: 0000-0003-4334-9547 


\section{Introduction}

Spot welding is a very frequently used technology for joining sheet metal components. The commercial relevance of spot welding is properly indicated by the demand of car industry, where several thousand spot welds per car are performed. But this technique is applied in many other areas of industry, too. It is a standard technology, which has been used in industry since the early 1940s. The quality of spot welding has a deterministic impact on the quality and on expected lifetime of machineries. Because of this, a quick and accurate inspection of each spot weld is very important to control quality in the safety and productivity of industrial products. There are standardized destructive methods, see e.g. [1], like chisel test, torsion test, tensile shear load test and the peel test. However, in general, the evaluation of spot welds by destructive tests needs high cost and long time and there are still significant error rates in the applied production processes.

To ensure all manufactured products have high quality, nondestructive testing methods are needed. Many reports of nondestructive techniques exist for spot welding such as ultrasonic [2-6] and radiographic methods [7]. Thermography [8,9] is another promising candidate to provide fast, contact-free and reliable testing of spot weld. It is nowadays widely used in NDT.

Different electromagnetic methods are also frequently used for inspection of spot welding. In [10] a real-time and in-situ monitoring method of spot welding is described, which takes the input electrical impedance of the welding system as the monitoring signature.

Eddy current testing (ECT) is also a widely and successfully used method for detection of surface or sub-surface defects of a metal [11]. ECT provides depth information due to the use of different frequencies. In order to survey the depth tips of defects, an ECT method using multiple frequencies was developed [12,13], where an analysis method for the internal structure of a spot weld by eddy current testing was developed. As a result, the correlation between these magnetic analyses of internal area and the strength of the spot weld was clarified [14].

Another area of nondestructive inspection of spot weld is based on magnetic methods. Compared with the ultrasonic and radiographic methods, the magnetic method does not provide good morphology information, however it is convenient and low-cost. A possible way to perform magnetic-based testing is the magnetic flux leakage test (MFL), which uses the magnetic flux leakage from the specimen surface when a low frequency magnetic flux is induced [15,16,17]. A nondestructive spot weld inspection method using MFL with a magnetoresistive sensor was reported recently [18]. A combined technique, using magnetic flux penetration and an eddy current testing was also developed [19].

Although there are no reports in the literature about application of magnetic hysteresis measurement methods for inspection of spot welding, this technique offers a lot of advantages, as well. Magnetic hysteresis is rather sensitive to any changes in the structure of the material, because magnetization processes are closely related to their microstructure [20,21], and welding process produces serious phase transformation in the welded area of the material. This makes this magnetic approach an obvious candidate for non-destructive testing in case of spot welding, too. Structural non-magnetic properties of ferromagnetic materials have been non-destructively tested using traditional hysteresis methods since long time with fair success. A number of techniques have been suggested, developed and currently used in industry, see e.g. [22]. They are mostly based on detection of structural variations via the classical parameters of major hysteresis loops. An alternative, more sensitive and more experimentally friendly approach to this topic was developed [23], which is based on systematic measurement and evaluation of magnetic minor hysteresis loops. This method, called Magnetic Adaptive Testing (MAT) introduces general magnetic descriptors to diverse 
variations in non-magnetic properties of ferromagnetic materials, optimally adapted to the just investigated property and material.

The purpose of the present work is to apply MAT for investigation of spot welding. The results, obtained on a system of weld spots in case of car-body sheets are shown, and the sensitivity and reliability of the method is also analyzed.

\section{Sample preparation}

A series of weld spots in car-body sheets was investigated. Two sheets were welded to each other by spot welding. The thickness of the sheets was $1.2 \mathrm{~mm}$, the material of the sheets was cold rolled low carbon steel (C content $0.1 \mathrm{Wt} \%$; Si $0.34 \mathrm{Wt} \%$; Ti $0.06 \mathrm{Wt} \%$; Mn 0.85 $\mathrm{Wt} \%$;). A series of welded spots (altogether twenty welded spots in a line) was produced by 2.4 bar pressure welding and the welding current was modified from 15.6 up to $21.8 \mathrm{kA}$. The size (diameter) of the welded spots is around $7 \mathrm{~mm}$. Some welded spots can be seen in Fig. 1.

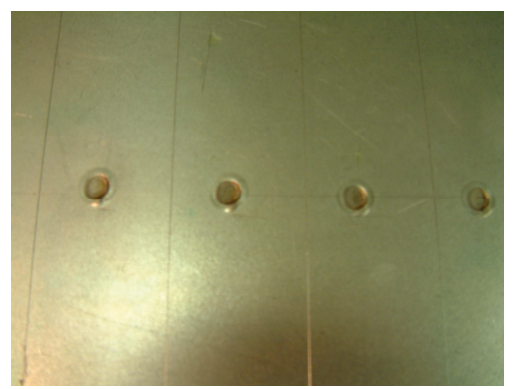

Fig. 1. Some welded spots (diameter is about $7 \mathrm{~mm}$ )

Destructive tests were also performed on a control series of welded spots, which can be seen in Fig. 2. Those welded spots, on which the magnetic measurement was performed, are situated in the middle of Fig. 2, while the upper part of the photo shows the welded spots (having the same property as the magnetically investigated ones), which were tested destructively by the peel test, i.e. by tearing the weld apart and measuring the size of the remaining weld. The result of this test confirmed, that the welded spots having the welding current between 18.5 and $20.2 \mathrm{kA}$ are considered as proper ones. The spots below this region were not welded strong enough, the points above $20.2 \mathrm{kA}$ were over-welded, where the material was damaged. The result of the destructive test can be better seen in Fig. 3 .

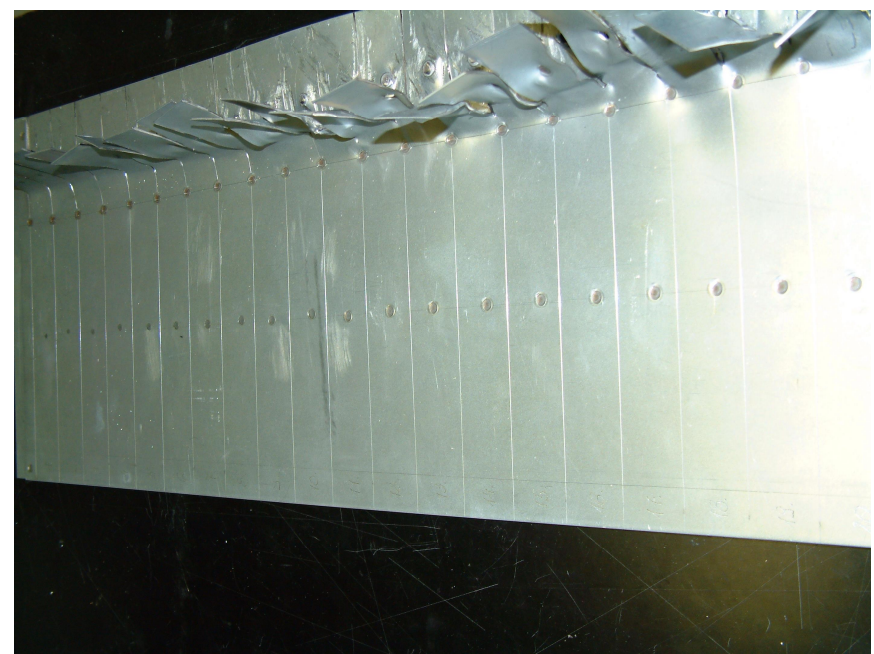

Fig. 2. Several welded spots, produced by different energy of welding. The upper part of the picture shows the result of the destructive test. 


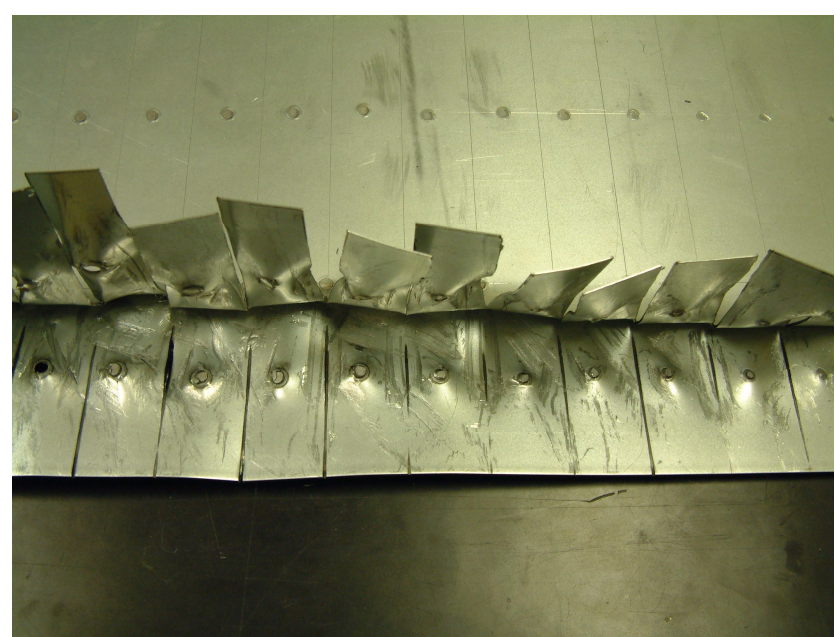

Fig. 3. Destructively tested welded spots

\section{Magnetic adaptive testing}

Magnetic adaptive testing was used for the magnetic measurements. MAT investigates a complex set of minor hysteresis loops (from a minimum amplitude of the magnetizing field, with increasing amplitude by regular steps) for each sample of the measured series. It follows from the theory of Preisach model of hysteresis [24], that such a set of experimental data contains complex information on hysteresis of the measured material.

The essential difference between material testing by the traditional hysteresis- and by the MAT-approach is shown in Fig. 4 schematically. The left hand part of the figure represents the traditional measurement of the single major (saturation) hysteresis loop. The major loop is measured for each of the investigated samples and the material degradation can be described through variation of values of any of the few major loop parameters, e.g. $H_{C}, B_{R}$, $\ldots$ as functions of an independent degradation variable, $\varepsilon$. The right hand part of the figure depicts schematically volume of the measured data for MAT. The large family of minor hysteresis loops is measured for each of the investigated samples and the material degradation can be then described through variation of values of any of the point (and/or slope) on any of the minor loops, i.e. $B\left(F_{i}, A_{j}\right)$ (and/or $\mu\left(F_{i}, A_{j}\right)$ ), as functions of any independent degradation variable, $\varepsilon$. In the present case the welding current, $I_{\text {welding, }}$, is the independent parameter, i.e. $I_{\text {welding }}$ values will be used later as $\varepsilon$.

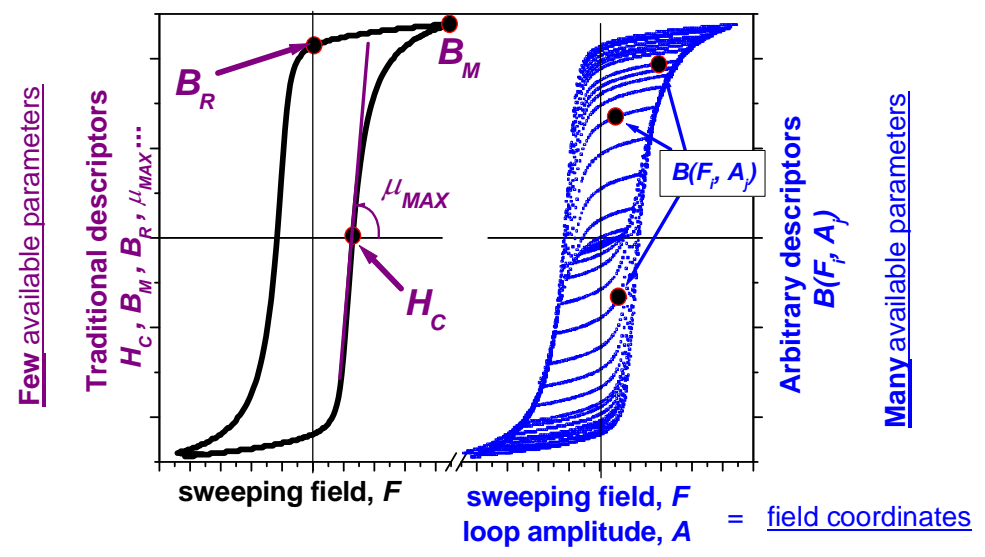

Fig. 4. Schematic comparison of the traditional magnetic hysteresis testing (left) and Magnetic Adaptive Testing (right). The traditional testing uses only a few parameters of the major loop for description of the material. Magnetic Adaptive Testing has the choice to pick up the best from many available parameters indexed by the field coordinates. 
The method of Magnetic Adaptive Testing utilizes systematic measurement of large families of minor hysteresis loops, from minimum amplitudes up to possibly the maximum (major) ones on degraded ferromagnetic samples/objects. From the large volume of the recorded data, those are applied for evaluation of the degradation, which reflect the material degradation in the most sensitive or otherwise the most convenient way. Such $і$ best adapted for the investigated case $\ddot{i}$ data are used as the MAT-parameter(s) and its / their dependence on an independent variable accompanying the inspected degradation is referred to as the MAT degradation function $(s)$.

The magnetic induction method appears to be the easiest way of the systematic measurement for MAT. A specially designed Permeameter [25] with a magnetizing yoke was applied for measurement of families of minor loops of the magnetic circuit differential permeability. The measurement of the hysteresis loops is performed by a magnetizing yoke, which is placed on the flat surface of the sample. The block-scheme of the device and the sketch of the yoke can be seen in Fig. 5a. The driving coil wound on the yoke produces triangular variations of the applied magnetic field with stepwise increasing amplitudes and with a fixed slope magnitude in all the triangles (see Fig. 5b).

The signal coil picks-up the induced voltage proportional to differential permeability of the sample. This triangular variation of the magnetizing field with time, $t$, and a voltage signal, $U$, is induced in the pick-up coil for each $k^{\text {th }}$ sample:

$$
U\left(\mathrm{~d} F / \mathrm{d} t, F, A_{j}, \varepsilon_{k}\right)=K^{*} \partial B\left(\mathrm{~d} F / \mathrm{d} t, F, A_{j}, \varepsilon_{k}\right) / \partial t=K^{*} \mu\left(\mathrm{d} F / \mathrm{d} t, F, A_{j}, \varepsilon_{k}\right) * \mathrm{~d} F / \mathrm{d} t,
$$

where $K$ is a constant determined by geometry of the sample and by the experimental arrangement. As long as $F=F(t)$ sweeps linearly with time $\ddot{i}$ i.e. $|\mathrm{d} F / \mathrm{d} t|$ is (the same) constant for measurement at each of the samples, Eq.(1) states, that the measured signal is simply proportional to the differential permeability, $\mu$, of the measured magnetic circuit, as it varies with the applied field, $F$, within each minor loop amplitude, $A_{j}$, for each $k^{\text {th }}$ measured sample. If we wish to get correct results without influence of any previous remanence, it is evident that each sample has to be thoroughly demagnetized before it is measured.

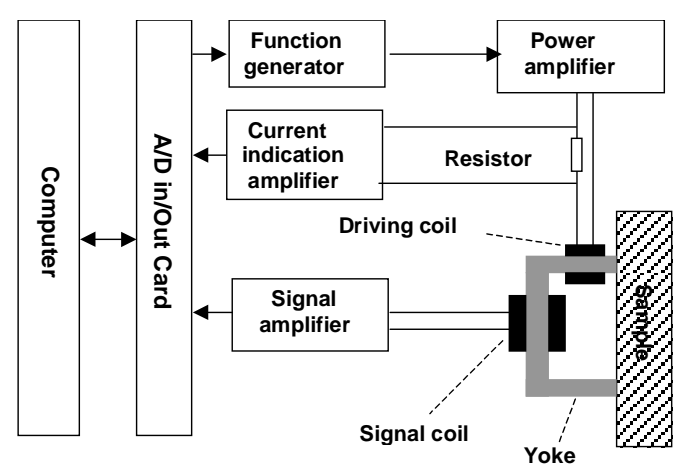

a)

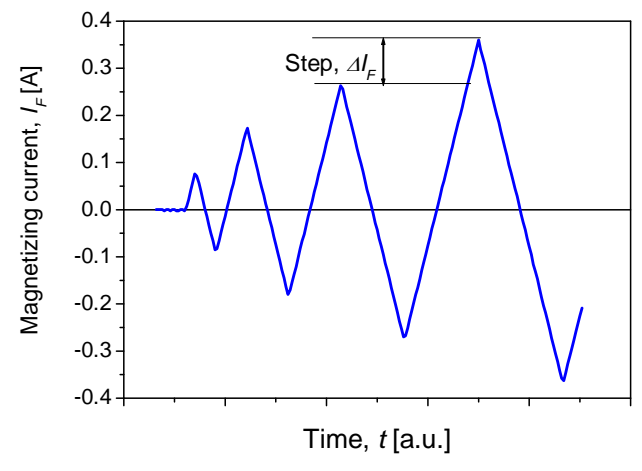

b)

Fig. 5. a) Block-scheme of the Permeameter and sketch of the yoke, b) Triangular variation of the magnetizing current with time.

The Permeameter works under control of a notebook PC, which sends the steering information to the function generator and collects the measured data. An input/output data acquisition card accomplishes the measurement. The computer registers actually two data files for each measured family of the minor $\mu$-shaped loops. The first one contains detailed information about all the pre-selected parameters of the demagnetization and of the measurement. The other file holds the course of the voltage signal, $U$, induced in the pick-up coil as a function of time, $t$, and of the magnetizing current, $I_{F}$, and/or field, $F$. As an 
illustration, Fig. 6 presents four families of permeability loops, measured on four different welded spots having different welding currents. Evidently it is a lot of data and our task is to compare them and to find the most suitable ones for characterizing the changes between samples.

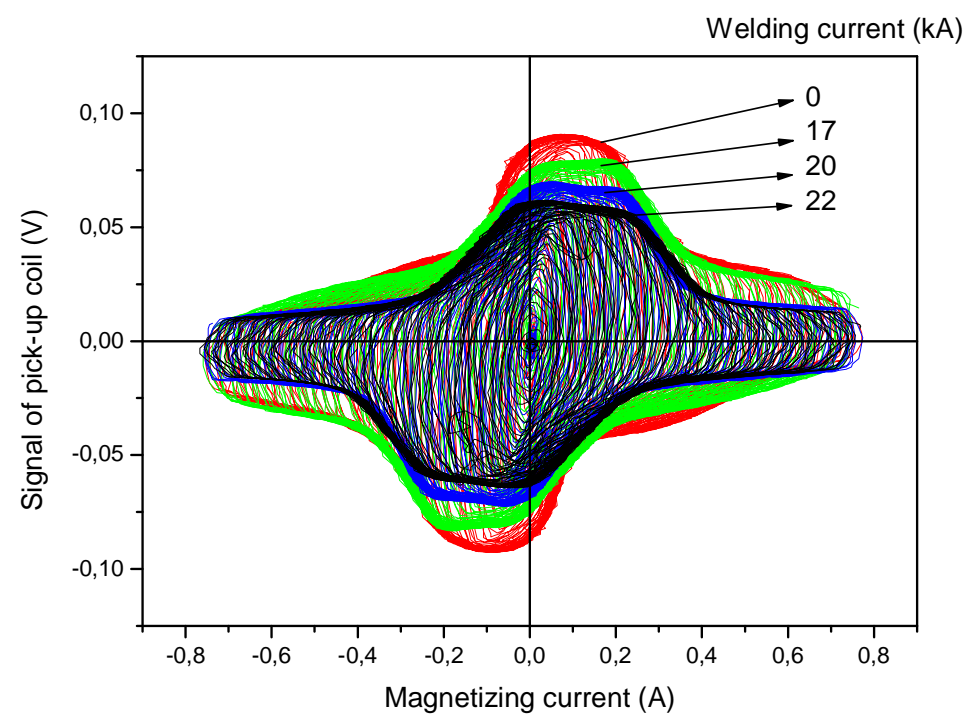

Fig. 6. Examples of families of the $\mu$-shaped loops vs. magnetizing current, measured on four different welded spots The positive and negative parts of the signal correspond to the increasing and decreasing parts of the triangular waveform of the current, respectively.

Instead of keeping the signal and the magnetizing field in shapes of continuous timedependent functions, it is practical to interpolate the family of data for each $\varepsilon_{k}$-sample into a discrete square $(i, j)$-matrix, $U\left(F_{i}, A_{j}, \varepsilon_{k}\right)$, with a suitably chosen step, $\Delta A=\Delta F$. (Because $\mathrm{d} F / \mathrm{d} t$ is a constant, identical for all measurements within one experiment, it is not necessary to write it explicitly as a variable of $U$.) MAT is a relative method (practically all the nondestructive methods are relative), and the most suitable information about degradation of the investigated material can be contained in variation of any element, of such matrices as a function of $\varepsilon$, relative with respect to the corresponding element of the reference matrix, $U\left(F_{i}, A_{j}, \varepsilon_{0}\right)$. So that we shall divide all $U\left(F_{i}, A_{j}, \varepsilon_{k}\right)$ elements by the corresponding elements $U\left(F_{i}, A_{j}, \varepsilon_{0}\right)$ of the reference sample matrix and obtain normalized elements of matrices of relative differential permeability $\mu\left(F_{i}, A_{j}, \varepsilon_{k}\right)=U\left(F_{i}, A_{j}, \varepsilon_{k}\right) / U\left(F_{i}, A_{j}, \varepsilon_{0}\right)$, and their proper sequences

$$
\mu\left(F_{i}, A_{j}, \varepsilon\right)=U\left(F_{i}, A_{j}, \varepsilon\right) / U\left(F_{i}, A_{j}, \varepsilon_{0}\right)
$$

as normalized $\mu$-degradation functions of the inspected material.

In some cases it turns out, that degradation functions of reciprocal values, such as $1 / \mu$ degradation functions are more convenient than the direct ones. Application of the reciprocal degradation functions proves effective especially in situations when $\ddot{i}$ with the increasing parameter $\varepsilon \ddot{i}$ the direct degradation functions approach kind of a ñsaturationò. Number of the degradation functions obtained from the MAT measurement depends on magnitude of the maximum minor loop amplitude, $A_{j}$, up to which the measurement is done, and on choice of the step value $\Delta A=\Delta F$ which is used for computation of the interpolated data matrices.

Once the degradation functions are computed, the next task is to find the optimum degradation function(s) for the most sensitive and enough robust description of the investigated material degradation. The matrix-evaluation program calculates also sensitivity 
of each permeability $\mu_{i j}(\varepsilon)$-degradation function and draws their ñsensitivity mapò in the plane of the field coordinates $\left(\mathrm{F}_{i}, \mathrm{~A}_{j}\right) \equiv(i, j)$. A 3D-plot of sensitivity of the degradation functions can substantially help to choose the optimum one(s). This map shows relative sensitivity of each $\mu_{i j}(\varepsilon)$-degradation function with respect to the independently measured $\varepsilon$-parameter of the investigated material. (In our case the $\varepsilon$-parameter is the strength of the welding current, as mentioned before, and will be used later.) Sensitivity of each degradation function is computed as the slope of its linear regression and it is expressed by a color and/or shade in the sensitivity map figure. This map is very useful if we want to characterize the reliability and reproducibility of the MAT descriptors. To ensure high enough sensitivity, MAT descriptors should be taken from the highest sensitive area. If area of the highest sensitivity is large enough, good reproducibility of the measurement is ensured. Map of relative sensitivity of the $1 / \mu\left(I_{\text {welding }}\right)$-degradation functions in the case of the spot welding will be shown in Fig. 9.

In our case it is not possible to determine the exact value of magnetic field inside the sample, due to the existing air gap between the yokeôs leg and the sample surface. Because of this the magnetizing current, $I$, (given in $\mathrm{mA}$ ) is used to characterize the samplesô magnetization when MAT descriptors are calculated. Because of this, on the following instead of $\mu\left(\mathrm{F}_{i}, \mathrm{~A}_{j}\right) \mu\left(\mathrm{I}_{i}, \mathrm{~A}_{j}\right)$ will be used, and current values will be given in $\mathrm{mA}$ units. The real value of magnetic field inside the sample is always proportional to the magnetizing current at a given arrangement, so this simplification does not cause any problem in the calculated (normalized) MAT descriptors.

The surface quality of the material to be measured and the air gap between the surface and magnetizing yoke is an extremely important factor in all magnetic measurements performed on open magnetic circuits. In our case these parameters are the same for all the measured spots. It means that correlation between the magnetizing current and the internal field is for all the measured spots really comparable.

\section{Results}

MAT measurements were performed on each welded spot with a magnetizing yoke equipped with a magnetizing and a pick-up coil (=measuring head). The yoke was a C-shaped laminated Fe-Si transformer core, placed on the sheet in such a way that the legs of the yoke were positioned outside the welded area. Lateral dimensions of the measuring head are characterized by its cross-section: $S=10 \times 4.5 \mathrm{~mm}^{2}$, the gap between the legs was $10.5 \mathrm{~mm}$. The magnetizing coil was wound on the bow of the yoke, with $N=150$ turns and the pick-up coil was wound on one of the yoke legs with $n=75$ turns. Fig. 7 shows the magnetizing yoke over a welded spot.

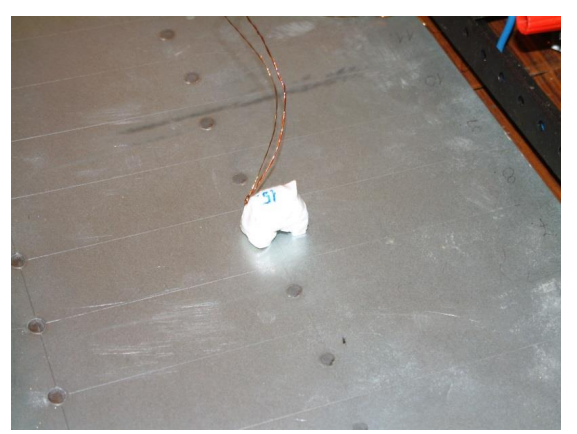

Fig. 7. MAT measurement: the measuring head (wrapped-up with a white protective tape) is positioned over a welded spot.

Results of the measurement are shown in Fig. 8, where the optimally chosen permeability degradation function vs. welding current can be seen. In order to obtain an 
increasing curve with a good sensitivity, the reciprocal, $1 / \mu\left(\mathrm{I}_{i}, \mathrm{~A}_{j}\right)$ degradation function was used. All the MAT degradation functions were normalized by the corresponding value of a reference spot (where no welding was located). To check the repeatability and reliability of the whole MAT measurement technique, the same welding procedure was made on two different car-body sheet systems (made of the same material with the same thickness) by using the same values of welding current. (The destructive test was performed only on one of the samples.) Fig. 8 contains the result of both measurements. As it is clearly seen in Fig. 8, very similar correlation was found in both cases. Scatter of measured points shows the error of the measurement.

More than $100 \%$ difference was found between the MAT degradation functions having the lowest and the highest energy of welding, which makes it possible to characterize easily quality of the welding.

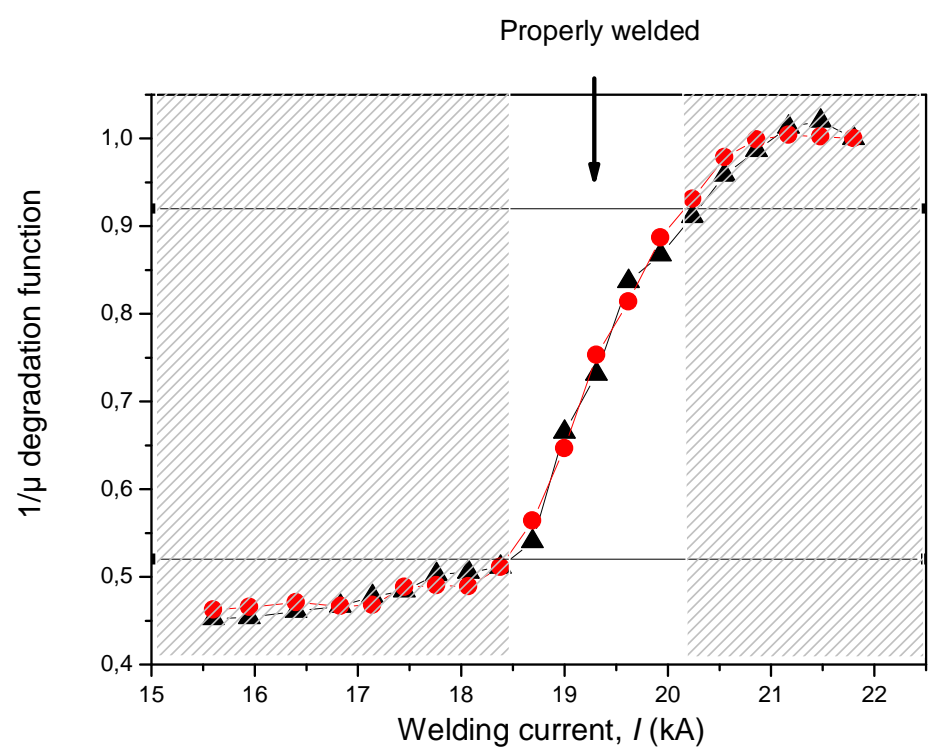

Fig. 8. The optimally chosen $\left(I_{i}=575 \mathrm{~mA}, A_{j}=700 \mathrm{~mA}\right) 1 / \mu\left(I_{i}, A_{j}\right)$ MAT degradation function vs. the welding current, measured on two series of welded spots, prepared by the same welding currents.

It is worth of mentioning that even a small change of the welding current results in a measurable modification in the MAT degradation functions at the beginning of the curve, and this becomes even more pronounced in the critical 18.5 and $20.2 \mathrm{kA}$ region. As it is shown in Fig. 8, the quality of the welded spot is considered acceptable, if the evaluated, normalized $1 / \mu\left(I_{i}, A_{j}\right)$ degradation function has a value between 0.52 and 0.92 .

The exact choice of the optimal degradation functions is not critical, the top sensitivity area of $1 / \mu$ degradation functions is very large. Fig. 9 shows the sensitivity map, and the crossing lines indicate the point, from where values of Fig. 8 were taken. As it follows from the presented sensitivity map, in Fig. 9, the most sensitive $1 / \mu$-degradation functions are those with field coordinates around $\left(I_{i}=575 \mathrm{~mA}, A_{j}=700 \mathrm{~mA}\right)$. The most sensitive $1 / \mu$-degradation functions are plotted in Fig. 8. However, if the degradation functions are taken from a relatively big area around the most sensitive point, marked by a rectangular in Fig. 9, the correlation between magnetic descriptors and welding current for all points within this area is very close (within the error of measurement) to each other. This ensures a very reliable and repeatable measurement. 


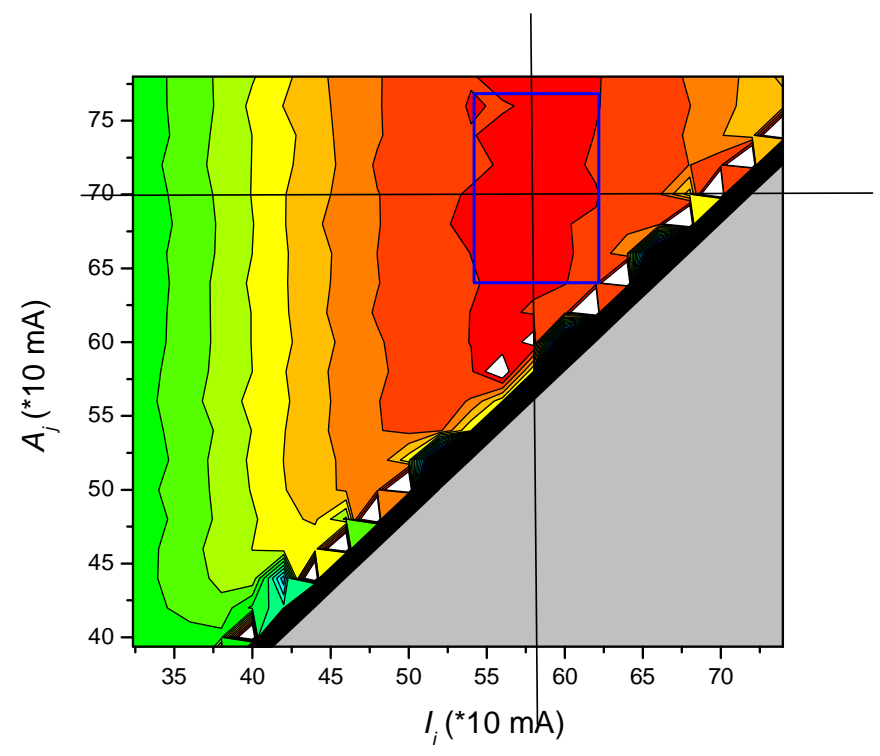

Fig. 9. Map of relative sensitivity of the $1 / \mu\left(I_{i}, A_{j}\right)$-degradation functions.

\section{Analysis of the possible error due to the measuring headô position}

If the MAT measurements are applied really at industrial environment, the tolerance towards the positioning of the measuring head is extremely important. We investigated this condition, too. The same welded spot was measured several times after each other, the position of the yoke with respect to the welded spot was modified, and the MAT evaluation was performed.

The result of the first series of measurement is seen in Fig. 10, where three characteristic cases are shown. In the first case the welded spot was positioned in the central area of the yoke (left in the figure). In the second case (middle in the figure) only a half area of the welded spot was under the yoke, while in the last case (right in the figure) the welded spot was completely outside. In this case the direction of yoke movement was perpendicular to the direction of the yoke axis. The figure shows how the $1 / \mu\left(I_{i}, A_{j}\right)$-degradation functions are changing compared with the central position of the yoke. In this case the MAT degradation functions were normalized by the corresponding one, which belongs to the central position of the yoke. It is seen that even in the most unfavorable case (which is a very improbable case in practice) only $3 \%$ difference in MAT degradation functions was experienced. 


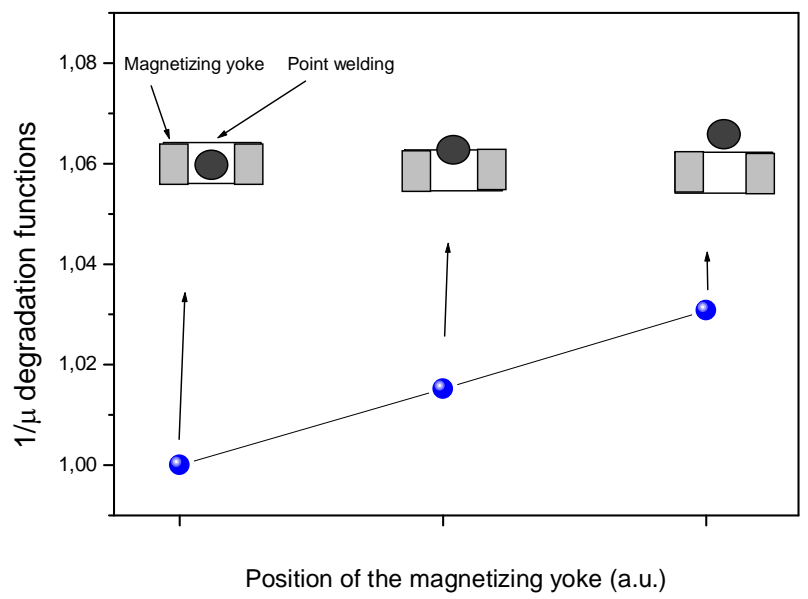

Fig. 10 The optimally chosen $\left(I_{i}=575 \mathrm{~mA}, A_{j}=700 \mathrm{~mA}\right) 1 / \mu\left(I_{i}, A_{j}\right)$ MAT degradation function, if the measurement was performed on the same welded spot at three characteristic positions of the yoke with respect to the welded spot, as indicated in the figure.

In the second series of similar measurements it was investigated, how the MAT degradation function is modified if the magnetizing yoke is positioned as indicated in Fig. 11. In this case the direction of yoke movement was parallel to the direction of yoke axis. As it is seen in Fig. 11, practically no difference between MAT descriptors was found in this case.

These results are considered as an excellent proof that MAT measurement is not sensitive to the precise positioning of the magnetizing yoke with respect to the position of the welding to be inspected.

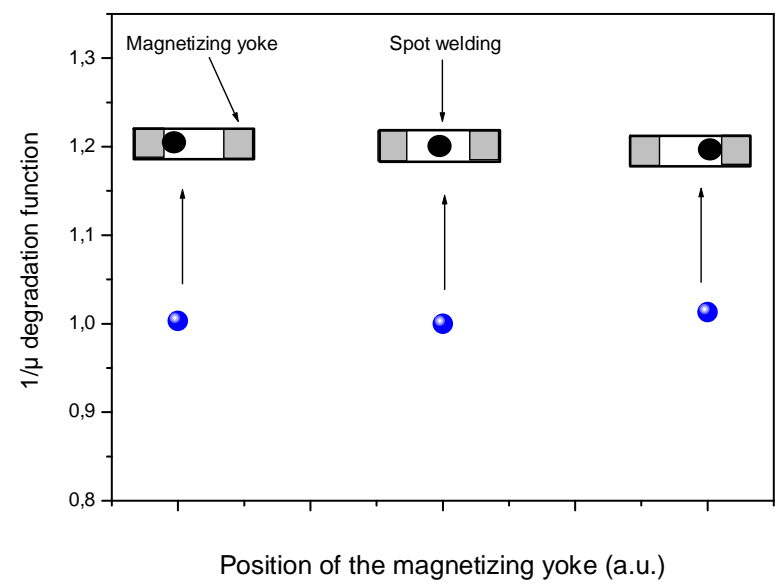

Fig. 11 The optimally chosen $\left(I_{i}=575 \mathrm{~mA}, A_{j}=700 \mathrm{~mA}\right) 1 / \mu\left(I_{i}, \mathrm{~A}_{j}\right)$ MAT degradation function, if the measurement was performed on the same welded spot at three characteristic positions of the yoke with respect to the welded spot, as indicated in the figure

\section{Conclusions}

The magnetic hysteresis method, Magnetic adaptive testing (MAT), which is based on systematic measurement and evaluation of minor hysteresis loops was applied for characterizing quality of spot welding. A series of welded spots, produced by different energy of welding current on car-body sheets was investigated. Good correlation was found between the value of the welding current $\ddot{i}$ which has a deterministic influence on the quality of 
welding $\ddot{i}$ and the magnetic descriptors. Based on this result, the spot welding can be characterized by a number, which correlates well with the result of a destructive test.

It was also shown that value of the magnetic descriptor determined by MAT is only very weakly sensitive with respect to the precise positioning of the magnetizing yoke and the welded point. This is promising for future possible practical application. The measurement is fast, simple and reliable.

\section{Acknowledgements}

The work was supported by the Hungarian Scientific Research Fund (project K 111662), and by the Czech Science Foundation (project No. 14-36566G) and by the Researcher Exchange Program between the Czech Academy of Sciences and Hungarian Academy of Sciences.

\section{References}

[1] DVS Merkblatt 2916-1: testing of resistance welded joints: destructive testing, quasi static

[2] E.T. Hall, D.I. Crecraft. Bonded joints and non-destructive testing. Nondestr. Test. 1971; 4: $181-191$

[3] L. Goglio, M. Rossetto. Ultrasonic testing of adhesive bonds of thin metal sheets. Nondestruct. Test. Eval. Int. 1999; 32: 323-331

[4] E. Blomme, D. Bulcaen, F. Declercq. Recent observations with air-cooled NDE in the frequency range of $650 \mathrm{kHz}$ to $1.2 \mathrm{MHz}$. Ultrasonic 2002; 40: 153-157

[5] Z.Chen, Y. Shi, B. Jiao, H. Zhao. Ultrasonic nondestructive evaluation of spot welds for zinc-coated high strength steel sheet based on wavelet packet analysis. J. Material Processing Technology 2009; 209: 2329-2337

[6] S. Vanlanduit. P. Guilaume, G. Van Der Linden. On-line monitoring of fatigue cracks using ultrasonic surface waves. NDT \& E International 2003;36: 601-607

[7] T.W. Liao. An automated radiographic NDT system for weld inspection. Nondestruct. Test. Eval. Int. 1998; 31: 183-192

[8] X. Malgadue. Theory and practice of infrared technology for nondestructive testing. Wiley, April 2001

[9] J. Schlichting, S. Brauser, L.A. Pepke, Ch. Maierhofer, M. Rethmeier, M. Kreutzbruck. Thermographic testing of spot welds. NDT\&E International 2012; 48: 23-29

[10] S.F. Ling, L.X Wan, Y.R. Wong, D.N Li. Input electrical impedance as quality monitoring signature for characterizing resistance spot welding. NDT \& E International 2010; 43: 200-205

[11] B.A. Auld, J.C. Moulder. Review of advances in quantitative eddy current nondestructive evaluation. J. Nondestruct. Eval. 1999; 18: 3-36

[12] Y. He, F. Luo, M. Pan, F. Weng, X. Hu, J. Gao, B. Liu. Pulsed eddy current technique for defect detection in aircraft riveted structures. NDT \& E International 2010; 43: 176181

[13] G.Y. Tian, SA. Sophian. Defect classification using a new feature for pulsed eddy current sensors. NDT \& E International 2005; 38: 77-82

[14] D. Harada, K. Sakai, T. Kiwa, T. Tsukada. Analysis of the internal structure of a spotweld by magnetic measurement. SINCE 2013, Singapore International NDT Conference \& Exhibition 2013, 19-20 July 2013

[15] K. Tsukada, M. Yoshioka, Y. Kawasaki, T. Kiwa. Detection of back-side pit on a ferrous plate by magnetic flux leakage method with analyzing magnetic field vector. NDT \& E International 2010; 43: 323-328

[16] J. Jun, J. Lee. Nondestructive evaluation of cracks in a paramagnetic specimen with low conductivity by penetration of magnetic fluid. NDT \& E International 2009; 42: 297-303 
[17] Y. Sun, Y. Kang, C. Qiu. A new NDT method based on permanent magnetic field perturbation. NDT \& E International 2011; 44: 1-7

[18] K. Tsukada, M. Yoshioka, T. Kiwa, Y. Hirano. A magnetic flux leakage method using a magnetoresistive sensor for nondestructive evaluation of spot welds. NDT \& E International 2011; 44: 101-105

[19] K. Tsukada, K. Miyake, D. Harada, K. Sakai, T. Kiwa. Magnetic nondestructive test for resistance spot welds using magnetic flux penetration end eddy current methods. J. Nondestruct. Eval. 2013; 2: 286-293

[20] H. Kronmüller, M. Fähnle. Micromagnetism and the Microstructure of Ferromagnetic Solids, Cambridge University Press, Cambridge, 2003.

[21] D.C. Jiles, Magnetic methods in nondestructive testing. K.H.J. Buschow et al., Ed., Encyclopedia of Materials Science and Technology, Elsevier Press, Oxford, (2001) p.6021

[22] J. Blitz, Electrical and magnetic methods of nondestructive testing, Bristol, Adam Hilger IOP Publishing, Ltd., 1991.

[23] I. Tomág Non-destructive magnetic adaptive testing of ferromagnetic materials. J.Magn.Magn.Mat. 2004; 268: 178-185

[24] I.D. Mayergoyz, Mathematical models of hysteresis, Springer-Verlag, New York, 1991

[25] I. Tomág O. Perevertov, Permeameter for Preisach approach to materials testing, JSAEM Studies in Applied Electromagnetics and Mechanics 9, Ed. Takagi T, Ueasaka M, IOS Press, Amsterdam, p. 5., 2001 